\title{
The expression of matrix metalloproteinase-13 is increased in vessels with blood-brain barrier impairment in a stroke-prone hypertensive model
}

\author{
Masaki Ueno ${ }^{1}$, Bin $\mathrm{Wu}^{1,2}$, Akira Nishiyama ${ }^{3}$, Cheng-long Huang ${ }^{4}$, Naohisa Hosomi ${ }^{5}$, Takashi Kusaka ${ }^{6}$, \\ Toshitaka Nakagawa ${ }^{7}$, Masayuki Onodera ${ }^{1}$, Mizue Kido ${ }^{1}$ and Haruhiko Sakamoto ${ }^{1}$
}

We previously reported that the blood-brain barrier (BBB) function was deteriorated in vessels located in the hippocampus, but not the cerebral cortex, in 3-month-old stroke-prone spontaneously hypertensive rats (SHRSP). Recently published data suggest that matrix metalloproteinase (MMP)-2 and MMP-9 play a critical role in the BBB disruption in stroke or cerebral ischemia. In this study, we examined gene and protein expressions of MMPs in the BBB-damaged hippocampal vessels of 3-month-old SHRSP, in the cerebral cortical vessels without BBB damage of SHRSP, and in the hippocampal and cerebral cortical ones without BBB damage of 3-month-old Wistar Kyoto (WKY) rats. The expressions of MMPs were examined by real-time quantitative reverse transcriptase-PCR (RT-PCR), western blotting and immunohistochemical techniques. The gene and protein expressions of MMP-13 were significantly increased in the hippocampal samples of SHRSP compared with samples without BBB damage, such as cerebral cortical samples of SHRSP or hippocampal samples of WKY. Immunostaining of MMP13 was seen in the cytoplasm of ED-1-positive perivascular cells in both rats and was colocalized with those of type IV collagen or osteopontin. The type IV collagen was also localized in the basement membrane. These findings indicate that the expression of MMP-13 is increased in BBB-damaged hippocampal vessels in hypertensive SHRSP compared with vessels without BBB impairment in normotensive WKY rats and may be involved in vascular remodeling.

Hypertension Research (2009) 32, 332-338; doi:10.1038/hr.2009.26; published online 20 March 2009

Keywords: blood-brain barrier; MMP-13; SHRSP

\section{INTRODUCTION}

A causative role of blood-brain barrier (BBB) impairment is suggested in the pathogenesis of vascular dementia with leakage of serum components from small vessels leading to neuronal and glial damage. ${ }^{1}$ To clarify the roles of BBB impairment in vascular dementia, we have examined $\mathrm{BBB}$ function in various conditions related to vascular dementia, such as hypertension, acute ischemia with reperfusion, chronic cerebral hypoperfusion and aging with or without memory deficits. $^{2-4}$ Consequently, among these animal models, the most localized BBB impairment was clearly seen in the hippocampus, but not the cerebral cortex, of 3-month-old spontaneously hypertensive rats (SHR), especially of 3-month-old stroke-prone SHR (SHRSP), ${ }^{4}$ which were established by Okamoto and $\mathrm{Aoki}^{5}$ and are described in detail elsewhere. ${ }^{6,7}$ It is known that the neuronal loss occurs with a reduction of gray matter volume in the CA1 subfield and dentate gyrus of the hippocampus in SHR at the age of 6 months or order, indicating that SHR can be a good animal model of vascular dementia. ${ }^{8,9}$ SHRSP is also well known as an excellent model for subcortical vascular dementia accompanied by small-vessel pathology, because these rats show structural alterations of small cerebral arteries, ${ }^{10}$ reduction in cerebral blood flow, ${ }^{11}$ white matter lesions ${ }^{12,13}$ and cognitive impairment. ${ }^{14,15}$ We then examined differences in gene expression between microdissected hippocampal vessels with BBB impairment in SHRSP and those without the impairment in Wistar Kyoto (WKY) rats using a microarray assay and reported that the expression of osteopontin, a matricellular protein, was increased in BBB-damaged vessels in hypertensive SHRSP compared with that in vessels without $\mathrm{BBB}$ impairment in WKY rats. ${ }^{16}$ The study also indicated that the immunoreactivity of osteopontin was present in perivascular ED1-positive macrophage/microglial cells and suggested the possibility that osteopontin might act as an inducible modulator of vascular remodeling, which likely restored BBB function in the damaged blood vessels. In addition, we recently reported ${ }^{17}$ that the expression of P-glycoprotein, a representative efflux transporter

${ }^{1}$ Faculty of Medicine, Department of Pathology and Host Defense, Kagawa University, Kagawa, Japan; ${ }^{2}$ Department of Gynecology and Obsterics, Second Affiliated Hospital of China Medical University, Shenyang, China; ${ }^{3}$ Faculty of Medicine, Department of Pharmacology, Kagawa University, Kagawa, Japan; ${ }^{4}$ Faculty of Medicine, Department of Surgery, Kagawa University, Kagawa, Japan; ${ }^{5}$ Faculty of Medicine, Department of Internal Medicine, Kagawa University, Kagawa, Japan; ${ }^{6}$ Faculty of Medicine, Maternal and Perinatal Center, Kagawa University, Kagawa, Japan and ${ }^{7}$ Life Science Research Center, Kagawa University, Kagawa, Japan

Correspondence: Associate Professor M Ueno, Faculty of Medicine, Department of Pathology and Host Defense, Kagawa University, 1750-1 Ikenobe, Miki-cho, Kita-gun, Kagawa 761-0793, Japan.

E-mail: masaueno@med.kagawa-u.ac.jp

Received 13 November 2008; revised 19 January 2009; accepted 21 January 2009; published online 20 March 2009 
in the brain vessels, was increased in vessels with BBB impairment in the hippocampus of SHRSP. However, it remains to be clarified how damaged BBB function is restored or modulated in the hippocampal vessels of SHRSP.

Matrix metalloproteinases (MMPs) are proteolytic enzymes and involved in the remodeling of the extracellular matrix in a variety of physiological and pathophysiological conditions. Among MMPs, MMP-1 is known as type I collagenase, whereas gelatinase A (MMP-2) and gelatinase B (MMP-9), type IV collagenases, are able to digest the endothelial basal lamina leading to the opening of the BBB. ${ }^{18-20}$ Expression of MMP-9 and MMP-2 has been shown to be significantly increased during stroke in human ${ }^{21}$ and animal models of focal ischemia. ${ }^{19,20,22,23}$ MMP-9 is of particular interest in the context of acute brain ischemia, because selective upregulation of MMP-9 has been observed in the brains of patients with stroke. ${ }^{24}$ Activation of MMP-9 with increased BBB permeability contributes to the early formation of vasogenic edema after focal cerebral ischemia. ${ }^{25,26}$ Direct intracerebral injection of one of the MMPs, MMP-2, caused an increase in capillary permeability and hemorrhage. ${ }^{18}$ In addition, it is known that MMP-2 plays a critical role in the BBB disruption, glial activation and white matter lesions after chronic cerebral hypoperfusion. ${ }^{27,28}$ In the rat model of chronic cerebral hypoperfusion, BBB function was disrupted in the white matter lesions. ${ }^{3}$ These results suggest that increased expression of MMP-2 and MMP-9 induce increased BBB permeability followed by brain damage. In addition, it was reported using an MMP protein array that high levels of MMP9 and MMP-13, also known as collagenase-3, are involved in diffusion-weighted image lesion growth. ${ }^{29}$ It remains to be clarified whether MMP-13 is involved in the BBB disruption.

In this study, we examined whether the expression of some MMPs increased in BBB-damaged vessels of hypertensive animal models.

\section{METHODS}

\section{Experimental animals and procedures}

The experimental protocols for animal care were in accordance with the National Research Council's Guidelines. Three-month-old male SHRSP/Izm $(n=14)$ and WKY/Izm $(n=14)$ rats (purchased from Japan SLC Inc., Hamamatsu, Japan) weighing $250-380 \mathrm{~g}$ were used. All efforts were made to minimize the number of animals used and their suffering. Systolic and diastolic arterial pressure was measured three times in each conscious rat by tail-cuff plethysmography (BP-98A; Softron Co., Tokyo, Japan) and the mean values were calculated. The animals were anesthetized with diethyl ether and then used for several experiments.

For real-time quantitative reverse transcriptase-PCR (RT-PCR) and western blot analyses, rats were perfused transcardially with $0.01 \mathrm{moll}^{-1}$ phosphatebuffered saline (PBS). Their brains were removed, quickly cut into several regions including the frontal cortex and the hippocampus and stored at $-80^{\circ} \mathrm{C}$. For immunocytochemical examination, rats were perfused transcardially with PBS and then with a fixative containing $4 \%$ paraformaldehyde in $0.1 \mathrm{moll}^{-1}$ phosphate buffer ( $\mathrm{pH}$ 7.4). The brains were removed and immersed in the same fixative.

\section{Real-time quantitative RT-PCR}

Total RNA was extracted from the hippocampus and the frontal cortex with Trizol, according to the manufacturer's instructions. The RNA concentrations were determined by spectroscopy at $260 \mathrm{~nm}$. Reverse transcription of total RNA $(5 \mu \mathrm{g})$ was performed with a hexa-deoxyribonucleotide mixture $(0.35 \mu \mathrm{g})$ as primer, and Moloney murine leukemia virus-reverse transcriptase and ribonuclease inhibitor (human placenta) at $37^{\circ} \mathrm{C}$ for $60 \mathrm{~min}$. The reaction was terminated by cooling at $70^{\circ} \mathrm{C}$ for $15 \mathrm{~min}$. To quantify gene expression, TaqMan real-time quantitative PCR was performed with the ABI PRISM 7700 Sequence Detection System using Assays on-Demand Gene Expression probes of MMPs (MMP-2, MMP-3, MMP-9 and MMP-13), or glyceraldehydes 3-phosphate dehydrogenase (GAPDH) and TaqMan Universal PCR Master Mix (Applied Biosystems, Foster City, CA, USA), as reported earlier. ${ }^{30}$ The PCR cycling conditions for all samples were as follows: $50^{\circ} \mathrm{C}, 2 \mathrm{~min}$ for AmpErase UNG activation; $95^{\circ} \mathrm{C}, 10 \mathrm{~min}$ for AmpliTaq Gold activation; and 50 cycles for melting $\left(95^{\circ} \mathrm{C}, 15 \mathrm{~s}\right)$ and annealing/extension $\left(60^{\circ} \mathrm{C}, 1 \mathrm{~min}\right)$ steps. ${ }^{16,31}$ Each sample was run in duplicate, and each PCR experiment included two nontemplate control wells. The comparative threshold cycle method (Applied Biosystems) was used to calculate gene expression in each sample relative to the value observed in control samples using GAPDH as a control for normalization among samples.

\section{Western blot analysis}

Western blot analysis was performed to evaluate the expression of various proteins. The brain tissues were homogenized in lysis buffer containing $10 \mathrm{mmoll}^{-1}$ Tris buffer containing $2 \mathrm{mmoll}^{-1}$ phenylmethylsulfonyl fluoride, and $10 \mathrm{mmoll}^{-1} \mathrm{~N}$-ethylmaleimide with a protease inhibitor. The protein concentration was determined with the Bradford reagent spectrophotometrically at $\mathrm{A}^{595}$. The extracts were solubilized in a Tris/glycine/sodium dodecyl sulfate sample buffer in the presence of 5\% 2-mercaptoethanol (Bio-Rad, Hercules, CA, USA) and heated at $95^{\circ} \mathrm{C}$ for $5 \mathrm{~min}$. Protein extracts $(40 \mu \mathrm{g})$ were then separated by $10 \%$ sodium dodecyl sulfate-polyacrylamide gel electrophoresis. Next, the separated proteins were transferred from gel to nitrocellulose membranes and blocked in a blocking solution (5\% dry milk in PBS) for $1 \mathrm{~h}$. Subsequently, each membrane was incubated with a primary antibody at $4^{\circ} \mathrm{C}$ overnight. The primary antibodies used in western blotting analysis were rabbit anti-MMP-1 (1:1000, Biomol, Plymouth Meeting, PA, USA), rabbit anti-MMP-2 (1:200, Santa Cruz, Santa Cruz, CA, USA), rabbit anti-MMP9 (1:200, Santa Cruz) and mouse anti-MMP-13 (1:200, Chemicon International, Temecula, CA, USA) antibodies. The membranes were then incubated with horseradish peroxidase-labeled antirabbit IgG or anti-mouse IgG antibody (1:5000 or 1:2000, Amersham Biosciences, Buckinghamshire, UK) for $1 \mathrm{~h}$ at room temperature. The proteins were visualized on enhanced chemiluminescence film (Hyperfilm; Amersham Biosciences) by applying them to a Western Blotting Detection System (Amersham Biosciences). Finally, the blots were reprobed using an antibody against $\alpha$-tubulin (1:100, Santa Cruz), followed by incubation in biotin-conjugated anti-mouse IgM antibody (1:500, Chemicon International) and then in horseradish peroxidase-conjugated anti-biotin antibody (1:1000, Cell Signaling, Danvers, MA, USA). Data are expressed as the relative differences among samples after normalization to $\alpha$-tubulin expression. The immunoreactive bands were analyzed with a LAS1000plus lumino-imaging analyzer (Fuji Film, Tokyo, Japan).

\section{Immunohistochemistry}

For triple staining, the tissue sections were treated with $10 \%$ normal serum and incubated with the mouse anti-MMP-13 antibody (1:200, Chemicon) or a goat anti-MMP-13 antibody (1:200, Chemicon) at $4^{\circ} \mathrm{C}$ overnight, followed by incubation in Alexa Fluor 488-conjugated anti-mouse IgG (1:200, Molecular Probes, Eugene, OR, USA) or in Rhodamin-conjugated anti-goat IgG antibody (1:200, Chemicon). After washing in PBS, the sections were incubated with rabbit anti-type IV collagen (1:100, Santa Cruz), rabbit anti-osteopontin antibody (1:200, Rockland, Gilbertsville, PA, USA) or mouse anti-ED1 antibody (1:200, Serotec Ltd, Oxford, UK) at $4{ }^{\circ} \mathrm{C}$ overnight, followed by incubation in Alexa Fluor 594-conjugated anti-rabbit IgG (1:200, Molecular Probes) or in fluorescein isothiocyanate-conjugated anti-mouse IgG antibody $(1: 200$, Vector laboratories, Burlingame, CA, USA). Cross-reactions were not detected after double staining. In addition, the sections were incubated for $20 \mathrm{~min}$ in 4',6-diamidino-2-phenylindole (DAPI, Molecular Probes) solution at room temperature, which was diluted to $400 \mathrm{~nm}$ in $1 \%$ albumin solution in PBS. The fluorescent signals were viewed under a confocal microscope (Bio-Rad Radiance 2100). As a control experiment, we performed an identical immunohistochemical procedure with omission of the primary antibody or using a normal serum instead of the primary antibody.

\section{Statistical analysis}

The values are shown as means \pm s.d. Statistical analysis was performed with two-way analysis of variance followed by the Tukey method. Differences were considered to be statistically significant when $P<0.05$. 


\section{RESULTS}

\section{Blood pressure}

Blood pressure was significantly higher in 3-month-old SHRSP $(210 \pm 14 / 174 \pm 12 \mathrm{~mm} \mathrm{Hg})$ than WKY rats $(131 \pm 10 / 101 \pm 10 \mathrm{mmHg})$ of the same age.

\section{Real-time quantitative RT-PCR analysis}

Real-time quantitative RT-PCR was performed to compare the quantity of gene expression of MMP-2, MMP-3, MMP-9 and MMP-13 among the hippocampal and cerebral cortical samples of SHRSP and WKY rats. MMP-2 gene expression was increased in hippocampal samples of SHRSP (1.5-fold) or WKY rats (2.2-fold) compared with cerebral cortical samples in rats of corresponding strains, although there were no significant differences in gene expression between rats of both strains (Figure 1a). There were no significant differences in MMP-3 gene expression among the samples in four groups (Figure 1b). MMP-9 gene expression was decreased in hippocampal samples of SHRSP (0.58-fold) or WKY (0.73-fold) compared with cerebral cortical samples in rats of corresponding strains, although there were no significant differences in gene expression between rats of both strains (Figure 1c). MMP-13 gene expression was increased in hippocampal samples of SHRSP compared with hippocampal samples of WKY (3.2-fold) or with cerebral cortical samples of SHRSP rats (2.5-fold) (Figure 1d).

\section{Western blot analysis}

Western blot analysis was performed to compare the quantity of protein expression of MMP-1, MMP-2, MMP-9 and MMP-13 among the hippocampal and cerebral cortical samples of SHRSP and WKY rats. There were no significant differences in MMP-1, MMP-2 and MMP-9 protein expression among samples in four groups (Figures 2a-c). Western blot analysis in MMP-13 expression showed that a major and a minor band were located at molecular weights of approximately $45-50$ and $20-25 \mathrm{kDa}$ (Figure $2 \mathrm{~d}$ ), respectively. The density in the minor band was increased in the hippocampal samples of SHRSP compared with the hippocampal ones of WKY rats (1.4-fold) (Figure 2d), although there were no significant differences in the density of the major band among samples in four groups (data not shown).

\section{Immunohistochemical analysis}

The immunostaining of MMP-13 was colocalized with that in the cytoplasm of ED1-positive perivascular cells in rats of both strains, indicating that MMP-13 is present in the cytoplasm of perivascular cells of microglia/microphage lineage (Figures $3 \mathrm{a}$ and $\mathrm{b}$ ). The immunostaining
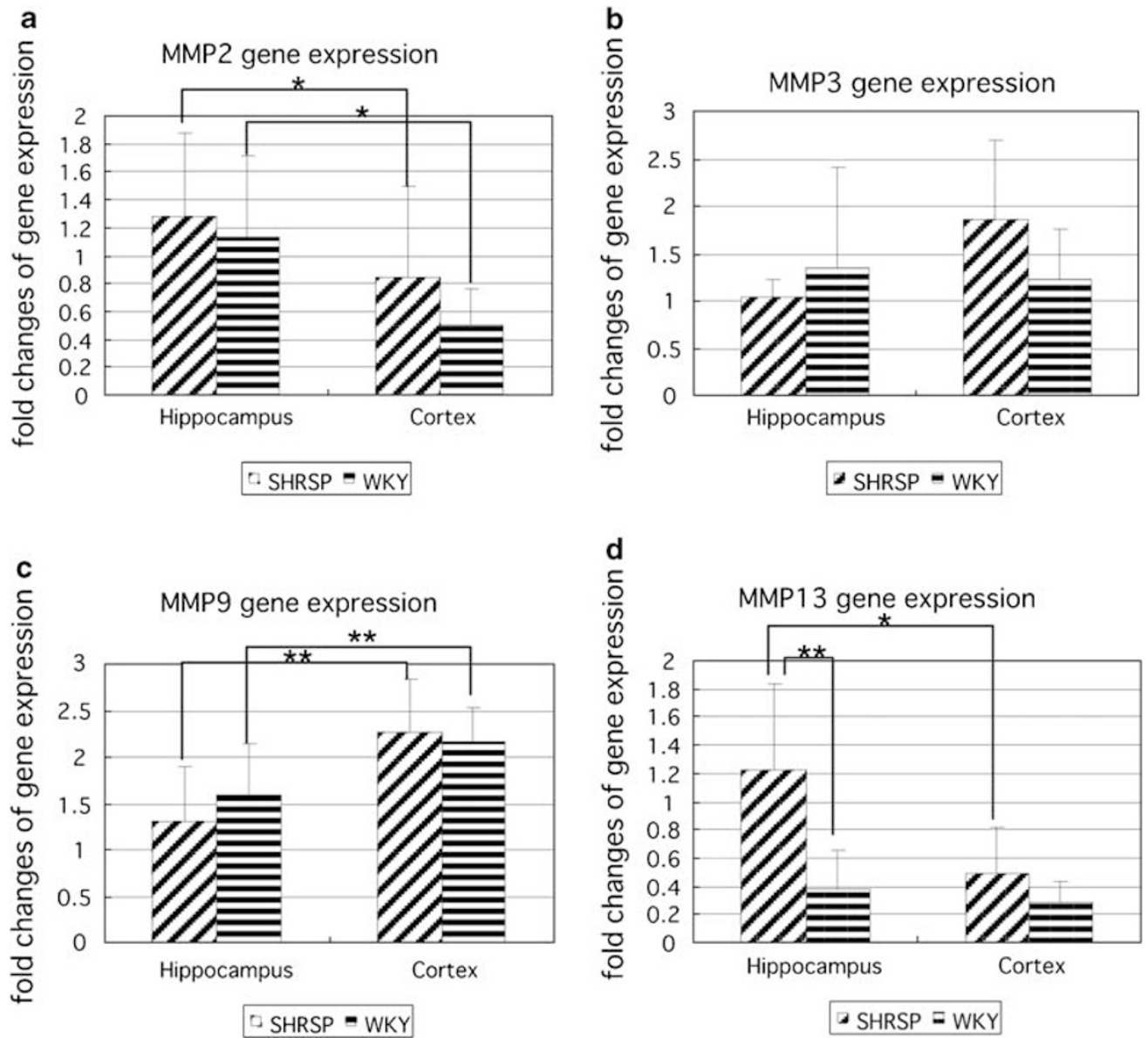

Figure 1 Fold changes of mRNA expression of matrix metalloproteinase-2 (MMP-2) (a), MMP-3 (b), MMP-9 (c) and MMP-13 (d) in the hippocampus and cerebral cortex of SHRSP and WKY rats. There are no significant differences in gene expression of MMP-2, MMP-3, MMP-9 between the same region in SHRSP and WKY rats, although regional differences in those of MMP-2 and MMP-9, but not MMP-3, are seen in SHRSP and WKY rats. MMP-13 gene expression is increased in the blood-brain barrier (BBB)-damaged hippocampal samples of SHRSP compared with samples without BBB damage, such as hippocampal ones of WKY or with cerebral cortical ones of SHRSP rats. Data are mean \pm s.d. $\left({ }^{*} P<0.05,{ }^{* *} P<0.01\right)$. 

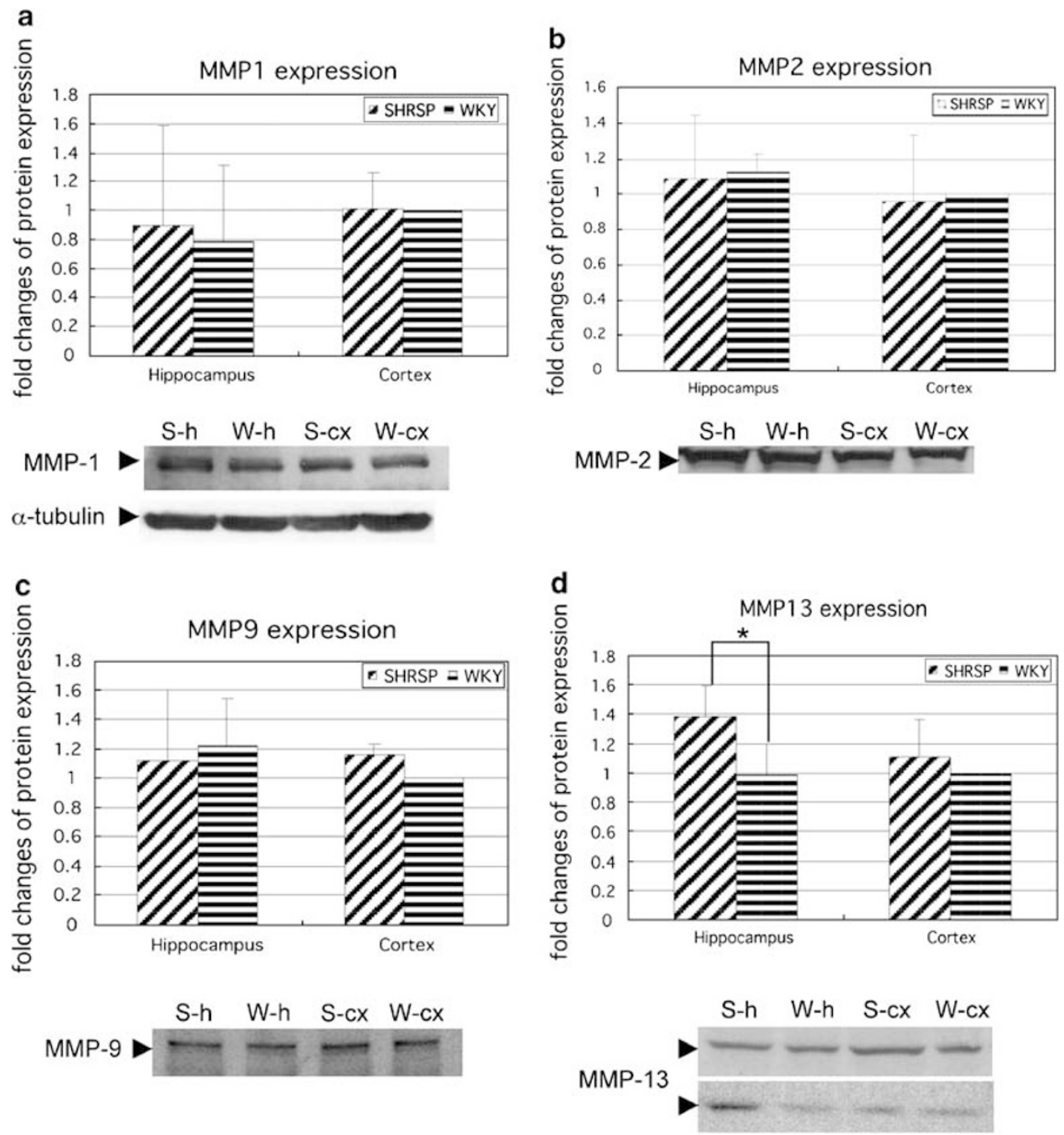

Figure 2 Western blot analysis of the expression of matrix metalloproteinase-1 (MMP-1) (a), MMP-2 (b), MMP-9 (c), MMP-13 (d) and $\alpha$-tubulin (a), and results of densitometric analysis of the MMPs immunoreactive bands in the hippocampus (S-h) and cerebral cortex (S-cx) of SHRSP and the hippocampus $(\mathrm{W}-\mathrm{h})$ and cerebral cortex (W-cx) of WKY rats (a-d). Data are expressed as the relative differences among samples after normalization to $\alpha$-tubulin expression. Major (upper side) and minor (lower side) MMP-13 immunoreactive bands, and analytic results of minor bands are shown in (d). The relative MMP-13 protein expression is better upregulated in hippocampal samples of SHRSP compared with hippocampal samples from WKY rats ( $\left.{ }^{\star} P<0.05\right)($ d). Data are mean \pm s.d.

of MMP-13 was also colocalized with that of collagen (Figures 3c and $\mathrm{d}$ ) or osteopontin (Figures $3 \mathrm{e}$ and $\mathrm{f}$ ). The type IV collagen was also localized in the basement membrane. There was no clear regional difference in the MMP-13 immunopositive structures between SHRSP and WKY rats (Figure 3).

\section{DISCUSSION}

The earlier study ${ }^{4}$ showed that the BBB function was deteriorated in vessels located along hippocampal fissures of 3-month-old SHRSP, but not in same-age WKY rats. The present study indicated that the gene and protein expression of MMP-13 were increased in hippocampal samples in SHRSP compared with those in WKY rats and that MMP13 was present in the cytoplasm of perivascular cells of microglia/ macrophage lineage. In this study, the immunostaining for MMP-13 was not clear in the endothelial cells of vessels. But, the role of MMP13 in the endothelial cells cannot be disregarded because MMP-13 in endothelial cells is known to play a role in protection of athero- sclerosis, ${ }^{30}$ which may affect barrier function. Nagel et al..$^{32}$ noted that two processed forms ( 47 and $20 \mathrm{kDa}$ ) of MMP-13 appeared in the brain after ischemia. These findings suggest that the MMP-13 expression is increased in the BBB-damage hippocampal vessels of SHRSP. Increased expression of MMP-13, also known as collagenase-3, may degrade the components of basement membranes of the hippocampal vessels, presumably followed by contribution to BBB damage.

We earlier reported ${ }^{4}$ that the $\mathrm{BBB}$ function was deteriorated in vessels located in the hippocampus, but not the cerebral cortex, in 3-month-old SHRSP. The reason for the regional difference is not clear. The hippocampus is supplied by the middle cerebral artery, especially proximal portions of it. It is thought that the middle cerebral arteries of SHR are proximal resistance arteries, exposed to major increases in blood pressure, and show increased resting tone and reduced arterial distensibility. ${ }^{33,34}$ Accordingly, it is likely that these factors affect BBB function in the hippocampus of hypertensive rats. Effects of genetic factors cannot be disregarded. 
SHRSP
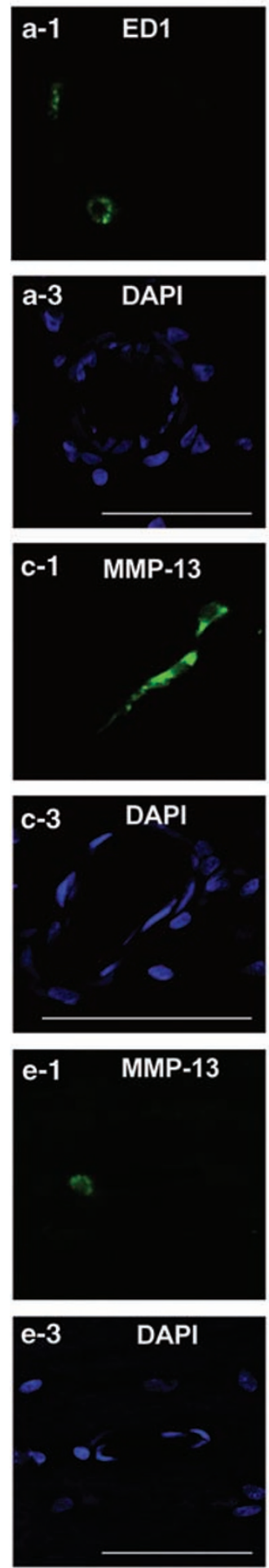
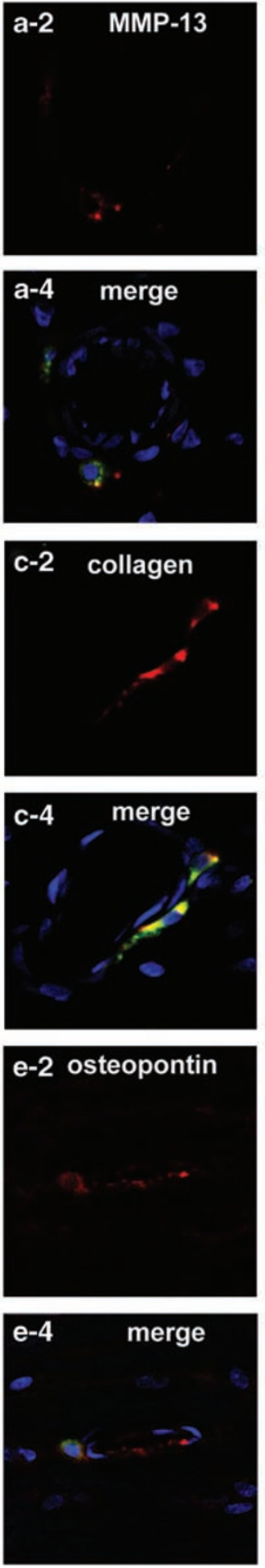
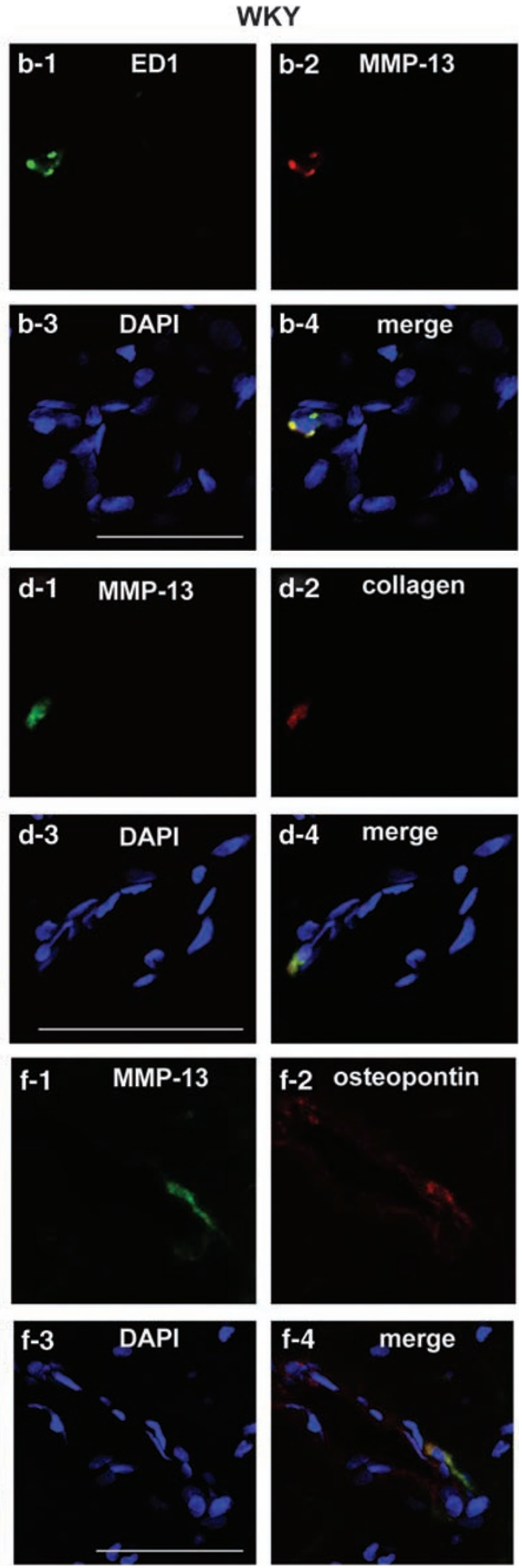

Figure 3 Confocal microscopic images of double immunofluorescent staining for the anti-MMP-13 antibody (visualized as red in a-2, b-2, or as green in c-1, d-1, e-1, f-1), the anti-ED1 antibody (visualized as green in a-1, b-1), or the anti-collagen antibody (visualized as red in c-2, d-2), or the anti-osteopontin antibody (visualized as red in e-2, f-2), the 4',6-diamidino-2-phenylindole (DAPI) staining (visualized as blue in a-3, b-3, c-3, d-3, e-3, f-3), and the overlapped images of triple staining $(\mathbf{a}-\mathbf{4}, \mathbf{b}-\mathbf{4}, \mathbf{c}-\mathbf{4}, \mathbf{d}-\mathbf{4}, \mathbf{e}-\mathbf{4}, \mathbf{f}-\mathbf{4})$ are shown. The coincidence of two kinds of antibodies is indicated by the conversion of green and red to yellow. Scale bars indicate $50 \mu \mathrm{m}$.

The earlier study ${ }^{4}$ also showed that collagen deposits were occasionally seen in the BBB-damaged vessels. In addition, Iwanaga et al. ${ }^{16}$ microdissected vascular samples from the hippocampus of SHRSP and
WKY rats and examined the difference in gene expression between the vascular samples with BBB impairment of SHRSP and those without impairment of WKY, using a microarray assay. Accordingly, 
osteopontin expression was increased in BBB-damaged hippocampal vessels in SHRSP, compared with hippocampal vessels without the BBB impairment in WKY rats. ${ }^{8}$ In this study, the expression of MMP-13 was colocalized with those of osteopontin as well as collagen, suggesting that MMP-13 expression is increased in BBB-damaged vessels with vascular collagenosis or remodeling. In addition, the results in the microarray assay also indicated that the expression of transforming growth factor- $\beta$ receptor II was increased in the hippocampal samples of SHRSP, compared with that in the samples of WKY rats. ${ }^{16}$ It is known that transforming growth factor- $\beta$ enhances MMP-13 expression. ${ }^{35,36}$ The increased expression of MMP-13 together with transforming growth factor- $\beta$ receptor II in the BBB-damaged vessels of SHRSP is compatible with these findings reported earlier. ${ }^{35,36}$

A matricellular protein, osteopontin, is expressed in response to mechanical stress and similar stimuli, integrates the inter-extracellular matrix signal transduction network of component cells, and maintains efficient contractility through quantitative and qualitative control of extracellular matrix proteins. ${ }^{37}$ In addition, osteopontin is reexpressed in the process of tissue damage and promotes myocardial remodeling through transforming growth factor- $\beta$ and MMPs. ${ }^{37}$ In tendon fibroblasts, MMP-13 expression can be downregulated by exogenous osteopontin. ${ }^{38}$ Accordingly, it is likely that increased expression of osteopontin in the hippocampal vessels of SHRSP rats may play a role in repair of BBB-damaged vessels with increased expression of MMP-13. Further studies are needed to clarify what accelerates the expression of MMP-13 or osteopontin in BBB-damage vessels. It remains to be clarified why only MMP-13 expression is increased in the BBB-damaged vessels of SHRSP rats among MMPs. It may be due to correlation to osteopontin. ${ }^{38}$ In addition, as angiotensin II and icotinamide adenine dinucleotide phosphate oxidase play a role in hypertensive brain injury of SHRSP, ${ }^{39}$ these may be related to hypertensive BBB damage.

\section{ACKNOWLEDGEMENTS}

We thank M Kawauchi for technical assistance and A Kimura for editorial assistance. This research was supported by a budget from the Ministry of Education, Culture, Sports, Science and Technology of Japan and a budget for priority research from Faculty of Medicine, Kagawa University, 2008.

\section{CONFLICT OF INTEREST}

The authors declare no conflict of interest.

1 Wardlaw JM, Sandercock PAG, Dennia MS, Starr J. Is breakdown of the blood-brain barrier responsible for lacunar stroke, leukoaraiosis, and dementia? Stroke 2003; 34: 806-812.

2 Ueno M, Sakamoto H, Kanenishi K, Onodera M, Akiguchi I, Hosokawa M. Ultrastructural and permeability features of microvessels in the hippocampus, cerebellum and pons of senescence-accelerated mice (SAM). Neurobiol Aging 2001; 22: 469-478.

3 Ueno M, Tomimoto H, Akiguchi I, Wakita H, Sakamoto H. Blood-brain barrier disruption in white matter of chronic cerebral hypoperfusion. J Cereb Blood Flow Metab 2002; 22 : 97-104.

4 Ueno M, Sakamoto H, Tomimoto H, Akiguchi I, Onodera M, Huang CL, Kanenishi K. Blood-brain barrier is impaired in the hippocampus of young adult spontaneously hypertensive rats. Acta Neuropathol 2004; 107: 532-538.

5 Okamoto K, Aoki K. Development of a strain of spontaneously hypertensive rats. Jpn Circ J 1963; 27: 282-293.

6 Okamoto K, Yamori Y, Nagaoka A. Establishment of the stroke prone spontaneously hypertensive rat. Circ Res 1974; 34/35: 143-153.

7 Hazama F, Ooshima A, Tanaka T, Tomimoto K, Okamoto K. Vascular lesions in the various substrains of spontaneously hypertensive rats and the effects of chronic salt ingestion. Jpn Circ J 1975; 39: 7-22.

8 Sabbatini M, Strocchi P, Vitaioli L, Amenta F. The hippocampus in spontaneously hypertensive rats: a quantitative microanatomical study. Neuroscience 2000; 100: 251-258.
9 Sabbatini M, Catalani A, Consoli C, Marletta N, Tomassoni D, Avola R. The hippocampus in spontaneously hypertensive rats: an animal model of vascular dementia. Mech Ageing Dev 2002; 123: 547-559.

10 Fredriksson K, Nordborg C, Kalimo H, Olsson Y, Johansson BB. Cerebral microangiopathy in stroke-prone spontaneously hypertensive rats. An immunohistochemical and ultrastructural study. Acta Neuropathol 1988; 75: 241-252.

11 Yamori Y, Horie R. Developmental course of hypertension and regional cerebral blood flow in stroke-prone spontaneously hypertensive rats. Stroke 1977; 8: 456-461.

12 Hazama F, Chue $\mathrm{CH}$, Kataoka H, Sasahara M, Amano S. Pathogenesis of lacuna-like cyst formation and diffuse degeneration of the white matter in the brain of stroke-prone spontaneously hypertensive rats. Clin Exp Pharmacol Physiol Suppl 1995; 22: S260-S261.

13 Lin JX, Tomimoto H, Akiguchi I, Wakita H, Shibasaki H, Horie R. White matter lesions and alteration of vascular cell composition in the brain of spontaneously hypertensive rats. Neuroreport 2001; 12: 1835-1839.

14 Saito H, Togashi H, Yoshioka M, Nakamura N, Minami M, Parvez H. Animal models of vascular dementia with emphasis on stroke-prone spontaneously hypertensive rats. Clin Exp Pharmacol Physiol Suppl 1995; 22: S257-S259.

15 Kimura S, Saito H, Minami M, Togashi H, Nakamura N, Nemoto M, Parvez HS. Pathogenesis of vascular dementia in stroke-prone spontaneously hypertensive rats. Toxicol 2000; 153: 167-178.

16 Iwanaga $\mathrm{Y}$, Ueno M, Ueki M, Huang CL, Tomita S, Okamoto $\mathrm{Y}$, Ogawa T, Ueda N, Maekawa N, Sakamoto $\mathrm{H}$. The expression of osteopontin is increased in vessels with blood-brain barrier impairment. Neurobiol Appl Neurobiol 2008; 34: 145-154.

17 Ueno M, Nakagawa T, Huang C, Ueki M, Kusaka T, Hosomi N, Kanenishi K, Onodera M, Wu B, Sakamoto H. The expression of P-glycoprotein is increased in vessels with bloodbrain barrier impairment in a stroke-prone hypertensive model. Neurobiol Appl Neurobiol 2009; 35: 147-155.

18 Rosenberg GA, Komfeld M, Estrada E, Kelly RO, Liotta LA, Stetler-Stevenson WG. TIMP-2 reduces proteolytic opening of blood-brain barrier by type IV collagenase. Brain Res 1992; 576: 203-207.

19 Rosenberg GA, Navratil M, Barone F, Feuerstein G. Proteolytic cascade enzymes increase in focal cerebral ischemia in rat. J Cereb Blood Flow Metab 1996; 16: 360-366.

20 Rosenberg GA, Estrada EY, Dencoff BS. Matrix metalloproteinsases and TIMPs are associated with blood-brain barrier opening after reperfusion in rat brain. Stroke 1998; 29: 2189-2195.

21 Anthony DC, Ferguson B, Matyzak MK, Miller KM, Esiri MM, Perry VH. Differential matrix metalloproteinase expression in cases of multiple sclerosis and stroke. Neuropathol App/ Neurobiol 1997; 23: 406-415.

22 Romanic AM, White RF, Arleth AJ, Ohlstein EH, Barone FC. Matrix metalloproteinase expression after cerebral focal ischemia in rats: inhibition of matrix metalloproteinase-9 reduces infarct size. Stroke 1998; 29: 1020-1030.

23 Chang DI, Hosomi N, Lucero J, Heo JH, Abumiya T, Mazar AP. Activation system for latent matrix metalloproteinase-2 are upregulated immediately after focal cerebral ischemia. J Cereb Blood Flow Metab 2003; 23: 1408-1419.

24 Maier CM, Hsieh L, Yu F, Bracci P, Chan PH. Matrix metalloproteinase-9 and myeloperoxidase expression: quantitative analysis by antigen immunohistochemistry in a model of transient focal cerebral ischemia. Stroke 2004; 35: 1169-1174.

25 Gasche Y, Fujimura M, Morita-Fujimura Y, Copin JC, Kawase M, Massengale J, Chan $\mathrm{PH}$. Early appearance of activated matrix metalloproteinase-9 after focal cerebral ischemia in mice: a possible role in blood-brain barrier dysfunction. J Cereb Blood Flow Metab 1999; 19: 1020-1028.

26 Fujimura M, Gasche Y, Morita-Fujimura Y, Massengale J, Kawase M, Chan PH. Early appearance of activated matrix metalloproteinase- 9 and blood-brain barrier disruption in mice after focal cerebral ischemia and reperfusion. Brain Res 1999; 842: 92-100.

27 Ihara M, Tomimoto H, Kinoshita M, Oh J, Noda M, Wakita H, Akiguchi I, Shibasaki H. Chronic cerebral hypoperfusion induces MMP-2 but not MMP-9 expression in the microglia and vascular endothelium of white matter. J Cereb Blood Flow Metab 2001; 21: 828-834.

28 Nakaji K, Ihara M, Takahashi C, Itohara S, Noda M, Takahashi R, Tomimoto H. Matrix metalloproteinase-2 plays a critical role in the pathogenesis of white matter lesions after chronic cerebral hypoperfusion in rodents. Stroke 2006; 37: 2816-2823.

29 Rosell A, Alvarez-Sabin J, Arenillas JF, Rovira A, Delgado P, Fernández-Cadenas I, Penalba A, Molina CA, Montaner J. A matrix metalloproteinase protein array reveals a strong relation between MMP-9 and MMP-13 with diffusion-weighted image lesion increase in human stroke. Stroke 2005; 36: 1415-1420.

30 Tarin C, Gomez M, Calvo E, Lopez JA, Zaragoza C. Endothelial nitric oxide deficiency reduces MMP-13-mediated cleavage of ICAM-1 in vascular endothelium. A role in atherosclerosis. Arterioscler Thromb Vasc Biol 2009; 29: 27-32.

31 Huang CL, Liu D, Kameyama K, Nakashima T, Yokomise H, Ueno M, Miyake M. MRP-1/ CD9 gene transduction downregulates Wnt signal pathways. Oncogene 2004; 23 : 7475-7483.

32 Nagel S, Sandy JD, Meyding-Lamade U, Schwark C, Bartsch JW, Wagner S. Focal cerebral ischemia induces changes in both MMP-13 and aggrecan around individual neurons. Brain Res 2005; 1056: 43-50.

33 Gonzalez JM, Somoza B, Conde MV, Fernandez-Alfonso MS, Gonzalez MC, Arribas SM. Hypertension increases middle cerebral artery resting tone in spontaneously hypertensive rats: role of tonic vasoactive factor availability. Clin Sci 2008; 114: 651-659.

34 Izzard AS, Horton S, Heerkens EH, Shaw L, Heagerty AM. Middle cerebral artery structure and distensibility during developing and established phases of hypertension in the spontaneously hypertensive rat. J Hypertens 2006; 24: 875-880. 
35 Johansson N, Ala-aho R, Uitto V, Grénman R, Fusenig NE, López-Otín C, Kähäri VM. Expression of collagenase-3 (MMP-13) and collagenase-1 (MMP-1) by transformed keratinocytes is dependent on the activity of p38 mitogen-activated protein kinase. J Cell Sci 2000; 113: 227-235.

36 Leivonen S-K, Chantry A, Hakkinen L, Han J, Kahari V-M. Smad 3 mediates transforming growth factor- $\beta$-induced collagenase- 3 (matrix metalloproteinase-13) expression in human gingival fibroblasts. J Biol Chem 2002; 277: 46338-46346.

37 Okamoto H. Osteopontin and cardiovascular system. Mol Cell Biochem 2007; 300: $1-7$.
38 Mori N, Majima T, Iwasaki N, Kon S, Miyakawa K, Kimura C, Tanaka K, Denhardt DT, Rittling S, Minami A, Uede T. The role of osteopontin in tendon tissue remodeling after denervation-induced mechanical stress deprivation. Matrix Biol 2007; 26: 42-53.

39 Yamamoto E, Tamamaki N, Nakamura T, Kataoka K, Tokutomi Y, Dong YF, Fukuda M, Matsuba S, Ogawa H, Kim-Mitsuyama S. Excess salt causes cerebral neuronal apoptosis and inflammation in stroke-prone hypertensive rats through angiotensin II-induced NADPH oxidase activation. Stroke 2008; 39: 3049-3056. 